\title{
Routing Protocols for Multi-hop Ad Hoc Networks: Operational Challenges and Design Optimizations
}

\author{
Prasanna J. Shete \\ Mumbai, India
}

\author{
R. N. Awale \\ Dept. of Electrical Engg., V.J.T.I \\ Mumbai, India
}

\begin{abstract}
Multi-hop Ad Hoc Networks are self-organizing networks characterized by dynamically changing topology due to node mobility and time varying characteristics of the wireless channel. Routing is a crucial issue in these networks. Several routing protocols have been proposed which fall into either of these categories: proactive, reactive or hybrid routing protocols. Proactive protocols have the advantage of less route establishment latency but suffer from heavy control overhead. Since routes maintained may never be used, system resources are unnecessarily wasted making proactive approaches less efficient. The reactive protocols overcome this drawback. These incur less overhead due to their "on demand" nature; nodes maintain routing information only when it is needed. Hence reactive protocols e.g AODV, DSR etc are preferred and widely adopted. But the on-demand behavior of these approaches itself leads to another problem, e.g the "broadcast storm" problem and thus challenges their usability. In this paper we highlight limitations and operational challenges of widely adopted reactive protocols and survey different optimization approaches suggested to overcome these challenges.
\end{abstract}

\section{General Terms}

Ad Hoc Networks

\section{Keywords}

MANETs, VANETs, DV, AODV, QoS.

\section{INTRODUCTION}

Ad Hoc Networks; MANETs, WSNs, VANETs etc. [1] are the networks of autonomous wireless devices that do not require any infrastructure. These are essentially multi-hop networks in which the source and destination may be many hops apart hence known as Multi-hop Ad Hoc Networks. They have gained popularity due to their low cost and ease of deployment and are useful in scenarios where infrastructure is impractical (e.g battlefields, mountainous terrain, remote locations) or is destroyed (due to disaster by natural calamity).

Multi-hop Ad Hoc Networks are characterized by dynamically changing topology due to high node mobility and time varying characteristics of the wireless channel as well as energy depletion of nodes. Thus routing is a crucial issue here. Varieties of routing protocols have been developed for ad hoc networks which can be broadly classified as: Proactive routing protocols, Reactive routing protocols and Hybrid routing protocols.

Proactive protocols are based on traditional wired routing protocols like Distance Vector or Link State Routing protocols. DSDV [2], WRP [3], OLSR [4] etc. are the widely accepted protocols from this category. These are table-driven protocols, in which each node maintains routing information for every other node of the network in "routing tables". The nodes periodically exchange routing table with their neighbors to maintain the topology. These protocols have the advantage of less or no latency in finding the path to destination, but suffer from large routing overhead. Also, they consume channel bandwidth as well as node battery. It may happen that, for maintaining routes which may never be used a lot of bandwidth is consumed and thus wasted. Also the periodic route updates consume the node energy and thereby reduce the node and/or network lifetime. Thus proactive protocols are less efficient and are useful in small networks with low node mobility.

Reactive protocols overcome these drawbacks of proactive protocols. These protocols maintain routes as and when required i.e. they operate "on-demand". Here a node discovers and maintains the routing info when it is actually required and not always. Thus the overhead is significantly less, but the latency in route establishment is quite large than proactive protocols. DSR [5], AODV [6], TORA [7] etc. are the widely used reactive routing protocols.

Hybrid routing protocols combine the pros of proactive and reactive routing approaches. The proximate node uses proactive routing based on routing table entries and far away nodes use on-demand discovery based approach. ZRP [8] is the most popular hybrid routing protocol.

Since the on-demand protocols (e.g DSR, AODV) have outperformed the proactive protocols they are adopted widely. Although these protocols reduce the routing overhead by operating "on demand" they give rise to another problem. Before data is to be delivered to a node for which no path is readily available, the source node has to "discover" it. The route discovery procedure relies on "flooding" of control packet called route request packets (RREQs) by source. Intermediate nodes between source and destination, which receive the request packets, are required to rebroadcast them. The transmission and retransmission of this special control packet consumes node energy as well as channel bandwidth. For a large network or in case of highly dynamic scenario where the routes changes are frequent this consumption is significant. Flooding of RREQs also results in "broadcast storm" problem [9] in the network.

This paper highlights the operational issues in proactive routing protocols in general and AODV in specific and surveys various optimization approaches suggested. The rest of paper is organized as follows: Section 2 elaborates the AODV mechanism, section 3 highlights the operational issues in AODV, survey of design optimization is given in section 4 and section 5 concludes the paper.

\section{AD HOC ON-DEMAND DISTANCE VECTOR ROUTING PROTOCOL}

In 1999 Perkins and Royer [6] proposed on-demand version of the DV routing protocol for ad hoc networks named AODV. AODV follows the notion of discovering the routes as and when required. Nodes maintain a route table for active routes as opposed to all possible routes for all the nodes, 
which consists next-hop routing information for destination. Each routing table entry has a lifetime associated with it. The route is considered valid only for the lifetime value. On expiry of this lifetime the routes are treated as invalid and are not used. AODV operation involves two phases; route discovery and route maintenance.

When a node has to send a data packet to a destination it checks its route table. If it does not have a valid entry in its route table for a destination, it starts the "route discovery" procedure. The source node uses a special control packet called route request (RREQ) packet which is broadcasted to all its neighbors. The RREQ consists of following fields:

<source addr; source sequence \#; broadcast id; destaddr; dest sequence \#; hopcnt>

The intermediate node on receiving the RREQ, checks whether it has route to destination, if route is available it replies with the route using route reply (RREP) packet; if no route is available the node simply re-broadcasts the RREQ. When RREQ is sent to next hop, a reverse path towards the source is set up. All next hop nodes follow a similar procedure till the destination is reached. i.e. Intermediate node floods the RREQ over the entire network. When RREQ reaches the destination, it generates the RREP which is propagated to the source following the reverse path. Forward path to destination is set when the RREP reaches the source. Nodes periodically exchange "Hello" packets to detect connectivity and maintain the routes. In case of link failure, the node sends an unsolicited RREP called RERR (route error) to all its active upstream neighbors with higher destination sequence number (1 greater than the current) and with infinity as hop count. This message ultimately reaches the source, and source comes to know about the failure. If source wants to discover a new route, it generates a new RREQ message with a larger destination sequence number.

\section{OPERATIONAL CHALLENGES IN REACTIVE ROUTING PROTOCOLS}

Although the reactive protocols try to reduce the routing overhead by operating "on demand" to overcome the drawbacks of proactive protocols, this on demand nature itself leads to another problem. When a data packet is to be delivered to a node for which no path is readily available, the source node discovers it. The route discovery procedure is based on flooding of route request packets (RREQs). The transmission of this special control packet consumes node energy and bandwidth. No doubt the size of request packet is small, but for a large network with many nodes where destination is many hops away from the source or in case of highly dynamic topology where the routes changes are frequent, this consumption is significant.

Moreover, in a multihop network scenario, route discovery procedure requires intermediate nodes to rebroadcast the route request packets originated from the source towards the destination. This rebroadcast of RREQs (flooding of RREqs) by intermediate node results in "RREQ storm" in the network [9]. It may happen that in a large network with many nodes, or a small network with high mobility, the entire channel bandwidth is occupied by these RREQs.

Moreover, in case of availability of multiple paths between source and destination, path selection is based on the criteria of shortest path and not the best path. The shortest path may be using weak links or links consisting energy critical nodes so it may happen that, the route reply (RREP) forwarded on this path may not reach the source, and the source after timeout has to re-initiate the route discovery.
Also, the speed and direction of the nodes, affects the performance of ad hoc routing protocols. The relative node mobility decides how long the link between the two nodes exists. The widely accepted reactive routing protocols like AODV or DSR have not taken into consideration these issues in their design and hence do not have any provision to solve them. Therefore it is necessary to optimize the routing protocols based on metrics like, Node energy, link quality, neighborhood node location knowledge, node velocity etc.

\section{OPTIMIZED ROUTING APPROACHES}

To optimize the routing mechanism of reactive protocols various approaches have been proposed that are based on the metrics discussed in section 3. We discuss some of these approaches on the grounds of principle, advantages and limitations in this section.

The optimization schemes discussed here fall into either of the following categories; probabilistic, energy-aware, location based, signal strength based or combination of these.

Gossiping based approach is suggested in [10] where each node forwards a packet with some probability. The RREQ broadcasts are controlled by controlling this probability. Source node sends a route request with probability 1 . When an intermediate node first receives a RREQ, it broadcasts it with a probability $p$, and discards it with probability $(1-p)$. The gossip based protocol saves up to $33 \%$ messages than flooding, for properly selected value of the probability. Gossiping also results in improved network performance in terms of end-to-end delay and throughput.

Gossiping improves the performance of denser networks or networks with high mobility, as the numbers of messages generated are quite large and thus percent saving achieved than flooding will be significant.

The key to gossiping performance is in the choice of gossip probability. It is quite tricky to select appropriate value of probability p. Also the number of messages saved by gossiping depends on various parameters like network topology, mobility and how frequently the messages are generated.

Limitation of gossiping is that it cannot find shortest routes. Routes found by gossiping may be 10 to $15 \%$ longer as compared to flooding. Thus in some cases, end-to-end delay will be more as compared with flooding. Also in sparse networks to establish connectivity, value of $\mathrm{p}$ should approach unity; else there may be holes in the network.

Neighbor node knowledge can be used to control the RREQ broadcast and thus conserve the resources. Neighbor coverage based probabilistic rebroadcast protocol (NCPR) for reducing routing overhead is proposed in [11]. Each node maintains its 1-hop neighborhood information in neighbor table. For effective exploitation of neighbor coverage knowledge a metric called "rebroadcast delay" is defined which is used (observed) by every node to determine the rebroadcast order and also to obtain additional coverage ratio i.e. ratio of number of nodes that should be covered by single broadcast to total number of neighbors. Connectively factor is used to maintain network connectivity at the same time, reduce the redundant transmissions. It determines how many neighbors should receive the RREQ packet. Combining additional coverage ratio and connectivity factor the "rebroadcast probability" is calculated which is used to reduce the number of rebroadcasts.

By combining the neighbor coverage knowledge and probabilistic mechanism the number of rebroadcasts are 
significantly reduced thus reducing the routing overhead. NCPR generates less rebroadcast traffic than flooding. Due to less redundant broadcast NCPR reduces network contention and collision thereby increases the PDR and reduces the endto-end delay.

The performance improvement of NCPR is significant in high density or heavy traffic networks. In sparse networks NCPR performance is slightly better than flooding. But at times the rebroadcast probability calculation exceeds 1 and is rounded to 1 , so NCPR behaves similar to flooding in such situations. Since the algorithm is quite complex it may consume more node energy. This paper does not discuss energy efficiency of NCPR. Although by using rebroadcast delay, efforts are made for speedy dissemination of RREQ to more number of nodes, but if these nodes are not part of the path converging to destination, the route setup delay may be large.

In [12] a protocol named DEARP (Distributed Energy Aware Routing Protocol) is suggested for wireless sensor networks. DEARP is essentially a modification to the well known AODV protocol. DEARP finds the routes in a decentralized fashion, taking into account energy efficiency and available energy in each node. Two metrics viz. energy cost and threshold are defined. These metrics are used to select energy efficient path and preserve the life of energy critical nodes and thus prolongs the network lifetime. Experimental results show that DEARP outperforms AODV on the grounds of battery power of individual nodes, network lifetime and throughput without causing increase in the delay.

DEARP selects energy efficient paths which lead to another drawback; the nodes along the selected path will rapidly lose their energy as all traffic is routed along the energy efficient path. Soon these paths will become unusable when the nodes become critical due to energy exhaustion. If initial energy of all nodes is same, the energy efficient paths selected may be paths with least number of longer hops. Longer the distance between nodes, more power will be required and hence more will be the energy consumption.

Moreover, energy constrained nodes are not allowed to participate in routing and hence challenge the network connectivity. If only one path exists to destination which includes energy critical node, such path will not be discovered and hence connectivity cannot be established.

For balanced energy consumption of nodes and thereby achieving prolonged network lifetime, an intelligent Reinforcement Learning (RL) based routing mechanism is suggested in [13]. The maximum lifetime routing problem is modeled as a RL problem. AODV protocol is extended with SARSA (i.e. State-Action-Reward-State-Action) RL mechanism, in which the mobile nodes use learning based adaptive energy efficient RREQ forwarding policy. The residual lifetime of mobile node in seconds, at time step $t$ is considered as a "state" $s_{t}$ calculated by taking the ratio of residual energy $\left(R E_{t}\right)$ and energy drain rate $\left(\right.$ DRate $\left._{t}\right)$ at time interval $t$. Each node monitors its energy consumption for $T$ second interval from which energy drain rate is calculated. High state value means the node has a high expected lifetime and low state value corresponds to node with poor expected lifetime. Based on the state value, the node performs action i.e. forwarding of RREQ. RREQ forwarding ratio is decided by picking up a probability from action set. Nodes with high state value forward more RREQs as compared with nodes having low state value. For the forwarding action a reward is calculated which is used for fair energy consumption among active nodes. Nodes with a lower energy drain rate will be rewarded with positive reinforcement and vice versa.
Results of simulation show that SARSA-AODV is more energy efficient as compared with AODV (modified with Residual Energy-aware Probability Model of Node), but incurs a large end-to-end delay. Since, RREQs carry the drain rate of all the nodes, size of RREQ increases with the size of network and thus the routing overhead.

Geographic routing identifies nodes by their locations and uses these coordinates to forward packets toward the destination in a greedy manner $[14,15]$. Geographical protocols are scalable since they only use localized neighboring information rather than complete network knowledge for next hop selection.

The routing overhead in reactive routing protocols increases due to link failures. To alleviate this limitation a Hybrid Location-based ad hoc routing protocol HLAR is proposed in [16]. HLAR uses the features of greedy geographic routing with reactive protocols and efficiently utilizes the location information to reduce the routing overhead. Also, when the location information is not accurate it uses the basic reactive routing mechanism and avoids the performance degradation caused due to location errors. In order to discover a route to destination, the source creates a route request (RREQ) packet that contains location of the source and destination node, it then consults its neighbor table to find if there existed any closer neighbor node towards the destination. If a closer node is available, RREQ is forwarded to that neighbor; if no closer neighbor is available RREQ is flooded to all neighbors. The intermediate nodes follow the same procedure in forwarding the RREQs. Also, the intermediate nodes involved in exchanging data traffic can locally repair broken routes using a route repair (RRP) packet rather than just reporting broken routes to its source.

The performance evaluation shows that the routing overhead rate of HLAR is constant for various node densities as compared with AODV, in which it grows exponentially with node density. The end-to-end delay is significantly less. Also, the PDR increases as a function of node density because large node density allows for easy route establishment and repair. The performance improvement of HLAR depends on the accuracy of location information. Though provision has been made to reduce the effect of location error, authors point that if this error is large, the performance improvement of HLAR will diminish. They also suggest that improvements to HLAR should be made to address service degradation caused due to effects like interference, longer distances between single hop nodes etc.

This paper does not comment about the effect of overhead due to including the location information in the RREQ packet. The local route repair mechanism may also delay the detection of route failures by the source, which may increase the delay.

A novel unicast protocol named, Cross Layer Weighted Position-based Routing (CLWPR) designed specially for VANETs in urban environments is proposed in [17]. CLWPR is a position based protocol, which uses the on-road distance as a metric for routing instead of Euclidean distance as is the case with most of location based schemes.

In VANETs the nodes are the vehicles travelling along the roads so the on-road distance between nodes will be more accurate than Euclidean distance in the forwarding decision. The proposed scheme also takes into account the PHY and MAC layer parameters like SNIR and MAC frame error rate to estimate the link quality. In addition queuing information is taken into consideration in terms of node utilization to provide traffic balancing for better QoS. This information is jointly 
used to calculate the "weight" for each neighboring node which is accounted in the forwarding decision.

In CLWPR there is no route discovery phase; rather it relies on 1-hop "Hello" messages that are periodically broadcast by every node. The Hello messages carry the position information (position, velocity and heading), MAC frame error rate and size of queue which is used by the nodes to calculate routing table.Before sending a packet, every node (source or intermediate), calculates its routing table. For each destination that the node has to send a packet it calculates a "weight" of every node in its neighboring list towards that destination. For sending the packets to a specific destination, that node is selected as the next hop node which has the minimum weight.

CLWPR demonstrates better performs in terms of packet delivery ratio and end-to-end delay as compared with GPSR. Since the "Hello" messages include lots of information, the traffic overhead is significant Authors claim this can be reduced by using position prediction to adjust the hello interval. However the prediction mechanism is not elaborated in this paper.

Estimated Distance-based Routing Protocol (EDRP) is proposed in [18] which restricts the forwarding range of RREQ messages in the direction of destination and thus reduces routing overhead. EDRP combines the features of position-based routing into on-demand routing protocols. An algorithm is proposed to estimate the distance, called EstD, between two nodes without positioning system like GPS

EstD is a combination of estimated geometrical distance (EGD) and estimated topological distance (ETD). EGD uses the variations in received signal strength (RSS) at contact time of two nodes to estimate future geometrical distance between them when they move apart. ETD is topology based EstD which refines EGD in case of inaccurate estimation. ETD is also based on RSS; RSS is used to estimate distance between neighboring nodes and by taking the sum of distance of every hop, the estimated distance between source and destination is calculated.

Using the EstD, the entire network area is divided into three zones: a) src-Zone; b) dst-Zone; and c) other-Zone. In each zone, a different probabilistic strategy is adopted to forward RREQ packets.

By propagating the RREQs in the direction of destination with the help of EstD the EDRP protocol significantly reduces the routing overhead and improves the routing performance. As can be seen from the performance evaluation, in EDRP normalized number of RREQ packets are decreased by $73.3 \%$ than AODV.

EDRP outperforms AODV in terms of packet delivery ratio and average end-to-end delay when the node density is large. Also EDRP outperforms AODV at all mobility speeds making it suitable for high mobility scenarios common in VANETs.

However, when node distribution is sparse, EDRP has poor performance than AODV. This is because when node density is sparse the possibility of "holes" in the network is large. Thus it is not suitable for sparse networks.

To overcome the broadcast storm problem of simple flooding, a Hybrid Flooding scheme is suggested in [19]. It combines the features of probabilistic, neighbor based and area based flooding schemes. Basically it is a probabilistic RREQ flooding scheme in which each node adjusts its forwarding probability based on neighborhood node density and the distance to the neighbors.

Node density metric named Expansion metric (E) is proposed. It defines the two-hop neighborhood density of a given node;
High value of $\mathrm{E}$ at an intermediate node indicates that its 2hop neighborhood is dense, whereas low value of $\mathrm{E}$ means it is not dense. This neighbor knowledge is used to control the probability of flooding the RREQs. In dense areas the RREQs should be flooded with low probability to reduce the redundancy of control packets, whereas in less dense areas RREQ flooding probability should be high. Also, a forwarding zone is defined (based on the node position info) which restricts the forwarding of received packets to the nodes which are located within it.

Nodes outside the zone do not forward the received packets. When an intermediate node located within the forwarding zone receives RREQ, it calculates its expansion metric and compares it with the value contained in the RREQ. Based on the result of comparison, the node calculates its forwarding probability.

The hybrid flooding scheme incurs less routing overhead as well as lesser energy consumption in dense network as compared to simple flooding or static probabilistic flooding.

For sparse networks the performance is comparable with simple flooding. Moreover this scheme is beneficial for nodes which are moving at speeds less than $36 \mathrm{~km} / \mathrm{hr}$, but as the speed increases the routing load increases. Thus this approach is not suitable for high mobility scenarios. e.g. VANETs.

BIO-inspired Cross-layer (BIOX) communication and coordination protocol for WSNs is proposed in [20]. It is a distance based approach inspired by the prey model in foraging theory.

Two nature-inspired profitability metrics are defined viz. nexthop selection and channel access. Next-hop selection profitability takes into account the node residual energy, whereas Channel access profitability deals with the congestion over a selected path.

Just like a forager searches for prey, each node searches for the possible next hop node using the next-hop selection profitability. The hop with maximum profitability in terms of residual energy is selected as next hop. Each sensor node periodically measures and broadcasts its residual energy to all of its neighbors. Thus, each sensor node knows the residual energy level of its neighbors. Sensor node $i$ will select that neighbor node as its next hop, which provides the maximum next-hop selection profitability. Thus using the next-hop selection profitability, each sensor node selects its next hop node in an energy-efficient way.

Channel access profitability allows each sensor node to obtain a sufficient level of packet transmission rate by regulating its channel access persistence. Based on the channel access profitability, each sensor node determines its transmission strategy during available time slots to forward its packets. BIOX enables balanced energy consumption of the nodes as well as minimizes the energy consumption throughout the network and thus prolongs the network lifetime. BIOX involves periodic broadcast of residual energy of nodes that will consume system resources. Also the profitability metric is based on thresholds, which may affect the network connectivity.

Reference [21] addresses the issue of alleviating the degradation of throughput due to link breakages caused by mobility. To reduce the packet loss due to mobility, a framework for interaction between physical, MAC, routing and transport layer is proposed. Based on the signal strength measurement at the physical layer, link management is performed. Proactive Link Management tries to predict link breakage while Reactive Link Management temporarily keeps the broken link alive with higher transmit power to keep alive the packets in transit. The AODV protocol is modified with 
addition of a route state called GoingDown (GDWN) state. GDWN state allows the nodes to salvage packets in transit. When link to the next hop starts getting weak, the modified AODV sends a GDWN packet to its active node, which is propagated to the source. The source stops sending packets and starts a new route discovery. The weaker link is kept alive temporarily by increasing the transmission power so as to salvage the packets in transit. Performance evaluation shows that, in high mobility scenarios the goodput of the TCP session is improved by $75 \%$ at light loads and $14-30 \%$ on average.

The proposed scheme monitors the link failures due to mobility alone ignoring the other causes of failures like congestion. Additional mechanisms will be required to monitor congestion in the network.

In scenarios of low mobility and high congestion, the temporary increase in transmission power will lead to adverse effect e.g. heavy congestion.

In reactive routing protocols, local link connectivity information is extremely important for route establishment and maintenance. Periodic beaconing of "Hello" messages is a widely-used scheme to obtain local link connectivity information. However, this periodic broadcast of Hello packets that are unnecessary consumes node energy and can drain batteries when mobile devices are not involved in active communication.

Reference [22] proposes an "Adaptive Hello messaging" scheme to suppress unnecessary Hello messages without risking detectability of broken links. The proposed scheme dynamically adjusts the Hello interval based on the node's involvement in communication (i.e sending or receiving of data). The average of time gap between two consecutive sending/receiving events, called the "event interval", is monitored to estimate how actively a node is involved in communication. The Hello interval is made proportional to this event interval. If the event interval is large, Hello interval is selected to be large and thus the unnecessary Hello messages are suppressed. When the node sends or receives a packet, the Hello interval is reset to default value.

The proposed approach limits Hello messages to half and thereby reduces the energy consumption of nodes to 54\% without any explicit difference in throughput and without incurring additional delay.

The performance evaluation shows that the effect of energy saving is high when the number of flows are less. As the number of flows increase, the energy saving decreases since more nodes will be involved in forwarding the packets and thus the Hello interval will be of default value.

Also due to longer Hello interval, the network connectivity may be affected. If a new node joins a network, it will be required to wait for a longer duration before it can acquire the neighborhood knowledge. Thus the route establishment delay will be increased.

The optimization approaches surveyed are summarized in Table1.

Table 1 Summary of Optimization Approaches

\begin{tabular}{|l|l|l|l|l|l|l|l|}
\hline Features $\rightarrow$ & Probabilistic & $\begin{array}{l}\text { Energy } \\
\text { Aware }\end{array}$ & $\begin{array}{l}\text { Signal } \\
\text { Strength } \\
\text { based } \\
\text { Routing } \\
\text { approaches }\end{array}$ & $\begin{array}{l}\text { Position/ } \\
\text { location/ } \\
\text { distance } \\
\text { based }\end{array}$ & $\begin{array}{l}\text { Local } \\
\text { Route } \\
\text { repair }\end{array}$ & $\begin{array}{l}\text { Connectivity } \\
\text { in sparse } \\
\text { Network }\end{array}$ & $\begin{array}{l}\text { Delay } \\
\text { (compared } \\
\text { with AODV) }\end{array}$ \\
\hline Gossip & Yes & No & No & No & No & Poor & More \\
\hline NCPR & Yes & No & No & No & No & Good & More \\
\hline DEARP & No & Yes & No & No & No & Poor & Comparable \\
\hline SARSA-AODV & Yes & Yes & No & No & No & Good & More \\
\hline HLAR & No & No & No & Yes & Yes & Good & Less \\
\hline CLWPR & No & No & No & Yes & No & Good & Less \\
\hline EDRP & Yes & No & Yes & Yes (Est) & No & Poor & Comparable \\
\hline Hybrid Flooding & Yes & No & No & Yes (Est) & No & Good & Comparable \\
\hline BIOX & Yes & Yes & No & Yes & No & Good & Comparable \\
\hline $\begin{array}{l}\text { Signal Strength } \\
\text { based Link Mgmt }\end{array}$ & No & No & Yes & No & No & Good & Comparable \\
\hline
\end{tabular}

\section{CONCLUSION}

Multi-hop wireless Ad Hoc Networks have gained acceptance due to their ad hoc capabilities. These networks are characterized by dynamically changing topology due to high mobility of nodes. Hence routing is a crucial issue in these networks. In this paper we have explored the routing protocols for multi-hop ad hoc networks with emphasis on reactive protocols. Operational challenges in widely accepted reactive routing protocols e.g. AODV are highlighted. Survey of various optimization approaches that attempt to overcome the 
limitations of existing protocols and achieve certain level of QoS is conducted and the features of these schemes are summarized.

\section{REFERENCES}

[1] D. Raychaudhuri and M. Gerla, "Emerging Wireless Technologies and the Future Mobile Internet", Cambridge University Press, 2011.

[2] C. E. Perkins, P. Bhagwat, "Highly Dynamic Destination-Sequenced Distance Vector Routing (DSDV) for Mobile Computers", Proceedings of ACM SIGCOMM 1994, pp. 234-244, Aug 1994.

[3] S. Murthy and J. J. Garcia-Luna-Aceves, "An Efficient Routing Protocol for Wireless Networks", ACM Mobile Networks and Applications Journal, Special Issue on Routing in Mobile Communication Networks, vol. 1, no. 2, pp. 183-197, Oct 1996.

[4] T. Clausen, P. Jacquet, A. Laouiti, P. Muhlethaler, A. Qayyum, and L. Viennot. "Optimized Link State Routing Protocol," in Proceedings of IEEE INMIC, Dec. 2001.

[5] D. B. Johnson, D. A. Maltz, "Dynamic Source Routing in Ad Hoc Wireless Networks", Mobile Computing, Kluwer Academic Publishers, vol. 353, pp. 153-181, 1996.

[6] C. E. Perkins, E. M. Royer, "Ad Hoc On-Demand Distance Vector Routing", Proceedings of IEEE Workshop on Mobile Computing Systems and Applications, pp. 90-100, February 1999.

[7] V. D. Park and M. S. Corson, "A Highly Adaptive Distributed Routing Algorithm for Mobile Wireless Networks", Proceedings of IEEE INFOCOM 1997, pp. 1405-1413, April 1997.

[8] M. R. Pearlman and Z. J. Haas. "Determining the Optimal Configuration for the Zone Routing Protocol." IEEE Journal on Selected Areas in Communications, Vol. 17 (8), 1395-1414, August 1999.

[9] O. Tonguz, N. Wisitpongphan, J. Parikh, F. Bai, P. Mudalige and V. Sadekar, "On the Broadcast Storm Problem in Ad hoc Wireless Networks", $3^{\text {rd }}$ Intl. Conf. on Broadcast Communications, Networks and Systems (BROADNETS), pp.1-11, Oct. 2006.

[10] Z. Haas, J. Y. Halpern, and L. Li, "Gossip-based Ad hoc Routing," IEEE/ACM Transactions on Networking, Vol. 14, No. 3, pp.479-491, Conference on Human Factors in Computing Systems, June 2006.

[11] X. M. Zhang, E.B. Wang, J. J. Xia and D. K. Sung, "A Neighbor Coverage based Probabilistic Rebroadcast for Reducing Routing Overhead in Mobile Ad hoc Networks", IEEE Transactions on Mobile Computing, Vol. 13, No.3, pp. 424-433, March 2013.
[12] M. M. Chandane, S.G. Bhirud and S.V. Bonde, "Distributed Energy Aware Routing Protocol for wireless Routing Protocol for Wireless Sensor Network", International Journal of Computer Applications (099758887), Vol. 34- No.3, pp. 6-11, Nov. 2011.

[13] Chettibi and S. Chikhi, "An adaptive Energy Aware Routing Protocol for MANETs using SARSA Reinforcement Learning Algorithm”, IEEE Conference on Evolving and Adaptive Intelligent Systems (EAIS), pp. 84- 89, May 2012.

[14] Y.B. Ko and N. H. Vaidya, "Location-Aided Routing (LAR) in Mobile Ad hoc Networks", Journal of Wireless Networks, No. 6, pp. 307-321, July 2000.

[15] B. Karp and H. T. Kung, "GPSR: Greedy Perimeter Stateless Routing for Wireless Networks", Proceedings of $6^{\text {th }}$ International Conference on Mobile Computing and Networking (MobiCom 2000), August 2000.

[16] M. A Rabayah and R. Malaney, "A New Scalable Hybrid Routing Protocol for VANETs", IEEE Transactions on Vehicular Technology, Vol. 61, No. 6, pp. 2625-2635, July 2012 .

[17] K. Katsaros, M. Dianati, R, Tafazolli and R. Kernchen, "CLWPR - A Novel Cross-Layer Optimized Position Based Routing Protocol for VANETs", Proceedings of IEEE Vehicular Networking Conference (VNC), pp. 139146, Nov. 2011.

[18] X. M. Zhang, E. B. Wang, J. J. Xia, and D. K. Sung, "An Estimated Distance based Routing Protocol for Mobile Ad hoc Networks," IEEE Transactions on Vehicular Technology, Vol.60, No.7, pp. 3473-3484, Sept. 2011.

[19] D. G. Reina, S. L. Toral, P. Johnson and F. Barreno, "Hybrid Flooding scheme for MANETs", IEEE Communications Letters, Vol, 17, No. 3, pp. 592-595, March 2013.

[20] B. Atakan, and O. B. Akan, "Bio-Inspired Cross-Layer Communication and Coordination in Sensor and Vehicular Actor Networks", IEEE Transactions on Vehicular Technology, Vol. 61, No. 5, pp. 2185-2193, June 2012.

[21] F. Klemm, Z. Ye, S.V. Krishnamurthy, and S.K. Tripathi, "Improving TCP Performance in Ad hoc Networks using Signal Strength based Link Management", Elsevier Journal on Ad Hoc Networks, Vol. 3, pp. 175-191, 2005.

[22] S. Y. Han and D. Lee, "An Adaptive Hello Messaging Scheme for Neighbor Discovery in On-Demand MANET Routing Protocols", IEEE Communication. Letters, Vol, 117, No. 5, pp. 1040-1043, May 2013. 